\title{
EVALUATION OF HOPKINSON BAR EXPERIMENTS USING MULTIPLE DIGITAL IMAGE CORRELATION SOFTWARE TOOLS
}

\author{
Marcel Adorna $^{a, *}$, Sttefan Bronder $^{b}$, Jan Falta $^{a}$, Petr Zlámal $^{a}$, \\ TOMÁŠ FÍlA ${ }^{a}$
}

${ }^{a}$ Czech Technical University in Prague, Faculty of Transportation Sciences, Department of Mechanics and Materials, Konviktská 20, 12000 Prague, Czech Republic

${ }^{b}$ Universität des Saarlandes, Institute of Applied Mechanics, Campus A4.2, 66123 Saarbrücken, Germany

* corresponding author: adorna@fd.cvut.cz

\begin{abstract}
Three different tools for Digital Image Correlation (DIC) were used for evaluation of dynamic experiments performed using custom Open Hopkinson Pressure Bar (OHPB) apparatus. High strain-rate measurements were performed on specimens of advanced cellular materials with predefined structure and negative Poisson's ratio. Low impedance polymethyl methacrylate (PMMA) bars instrumented with foil strain-gauges were used for dynamic loading of the specimens. Experiments were observed using a pair of high-speed cameras for imaging of loading process in sufficient quality. Custom developed evaluation DIC tool implemented in Matlab, open-source Matlab tool (NCorr) and commercial DIC software (ISTRA 4D) were all used for evaluation of image sequences recorded by high-speed cameras. Comparison of results obtained using all three different DIC tools and results of complementary strain-gauge measurement are shown in this paper. Verification of reliability of custom made DIC software tool is presented.
\end{abstract}

KeYwords: Digital Image Correlation, Hopkinson Bar, impact loading, high-speed imaging.

\section{INTRODUCTION}

Digital Image Correlation (DIC) is a well established method used for contactless assessment of full-field maps of displacement and strain on the surface of the deformed specimen. It shows a great promise for evaluation of high strain-rate experiments performed using Hopkinson Bar experimental device with specimens of geometrically complex structures [1].

In this study, three different DIC software tools were used for evaluation of direct impact Open Hopkinson Presure Bar (OHPB) experiments performed on specimens of advanced materials with predefined structure and negative Poisson's ratio (so called auxetic structures). The compared DIC softwares were: i) custom developed DIC tool implemented in Matlab [2, ii) open-source Matlab tool NCorr $[3$ and iii) commercial DIC software ISTRA 4D. Full-field DIC analysis of the specimens surfaces was performed using all three software tools with similar parameters settings. Obtained results were compared visually and quantitatively. Results were also compared with complementary strain-gauge measurement. Correlation of single nodal point of the specimen structure was also performed and influence of correlation coefficient was discussed.

\section{MATERIALS AND METHOdS}

\subsection{SPECIMENS}

The specimens geometry was based on a periodical assembly of $3 \times 3 \times 3$ re-entrant honeycomb auxetic unit cells. The specimens were printed using the Pro
Jet HD3000 3D printer (3D Systems, Rock Hill, USA) from the UV curable polymer VisiJet EX200 at the highest available printing resolution $(656 \times 656 \times 800$ DPI). This polymeric core samples were dip coated with a thin layer of carbon and an electrodepostion of nanocrystalline nickel $(\mathrm{Ni})$ was subsequently carried out, resulting in a homogeneous layer of nickle (coating thickness of approx. $60 \mu \mathrm{m}$ and $120 \mu \mathrm{m}$ ) within the whole structure. Finally the core polymer structure was melted out of the specimens using pyrosis at approx. $1000{ }^{\circ} \mathrm{C}$. For more information regarding the used specimens manufacturing process see [4. The specimens of the 3D printed nickle coated structures are shown in Fig. 1. The overall sample dimensions (selected specifically to suit the dimensions of the OHPB apparatus) of the specimens were approximately $13 \times 13 \times 19 \mathrm{~mm}$.

\subsection{Digital Image Correlation}

High-speed imaging enables to capture the deformation process of the OHPB tests in a suitable quality (sufficient number of images with small changes between them can be captured during experiment) for evaluation using optical methods. DIC method enables for the evaluation of strain fields on the surface of geometrically complex structures by tracking of a virtual pattern selected in the sequence of images where the displacements are to be evaluated. Each point of the virtual pattern is subsequently tracked through the whole captured image sequence. The tracking algorithm is based on subsets of pixels which are defined as an area with specific pixel dimensions formed 


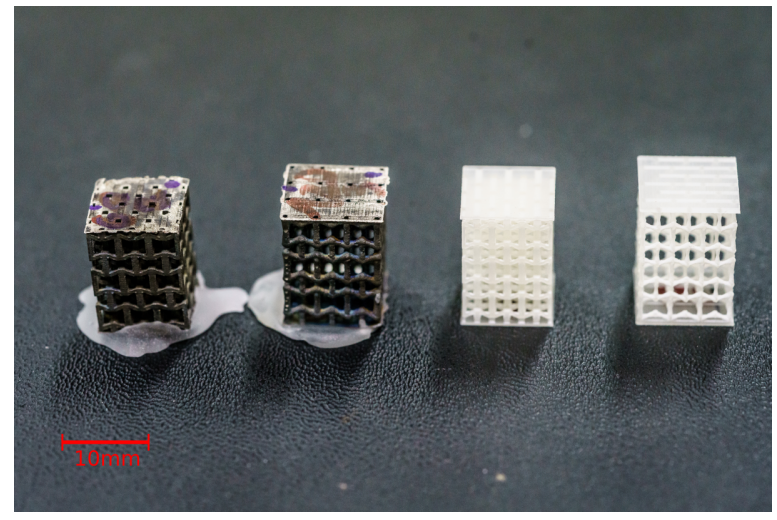

FiguRE 1. Specimens of 3D printed nickle coated structure (left) and the uncoated structure (right)

around the individual correlation point (Fig. 2). As the specimen is deformed, the reference pattern is deformed as well in the image sequence. The new position of the deformed subset is determined based on the extreme value of a correlation coefficient calculated using the selected criterion. Note, that not the whole area of the deformed picture is searched, but only an offset area, which reduce the computational complexity.

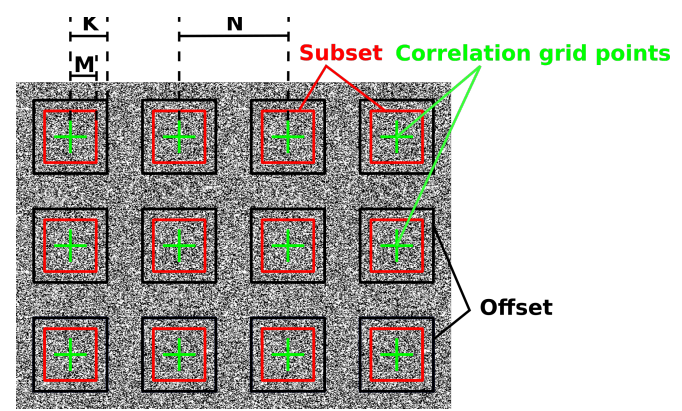

Figure 2. Parameters of the Digital Image Correlation, $\mathrm{M}$ - subset dimension, $\mathrm{K}$ - offset dimension, $\mathrm{N}$ grid spacing

\subsubsection{Custom DiC Matlab tool}

In our department, a custom developed DIC Matlab tool is commonly used for evaluation of various experiments. It is based on the Lucas-Kanade tracking technique, the correlation coefficients are estimated during a two-step procedure, first at the pixel level and second at sub-pixel level to determine the displacement with a higher precision. At the pixel level, the sum-squared difference (SSD) criterion is used whereas at the sub-pixel level the Lucas-Kanade algorithm based on the zero-normalised SSD (ZNSSD) criterion is used [5].

\subsubsection{NCORR MATLAB TOOL}

Ncorr is an open source 2D DIC Matlab program, which implements several different correlation procedures and criterions. However, for the evaluation of OHPB measurements, only one of the procedures provided results comparable with our custom- developed Matlab tool. In this procedure the zeronormalized cross correlation criterion (ZNCC) in combination with the Gauss-Newton nonlinear iterative least squares method is used.

\subsubsection{ISTRA4D}

ISTRA4D is a very robust commercial multi-camera DIC software. It implements many optimized correlation procedures, however the user is not able to select them manually and is therefore not in a full control of the correlation procedure. Correlation criteria and their inner parameters are not accessible or modifiable. Results obtained using ISTRA4D are compared with other software tools, however, precision and reliability of ISTRA4D software is not discussed in this paper.

\subsection{Open Hopkinson Pressure Bar}

High strain-rate experiments with the auxetic specimens were performed using the OHPB direct impact apparatus [6]. In contrary to conventionally used Split Hopkinson Pressure Bar (SHPB) technique, in the OHPB no striker bar is used and instead an instrumented incident bar is accelerated by a gas-gun system and directly hits a specimen mounted at the end of a transmission bar in the setup. In our OHPB setup, the incident bar is guided by a gas-gun barrel and by a low friction linear guidance system. This apparatus can be used to compress specimens to a considerably high strain. Moreover, as both the incident and the transmission bar are instrumented with strain-gauges, it is possible to evaluate the data from both contact faces of the mounted specimen. Due to a low mechanical impedance of the tested specimens, the polymethyl-methacrylate (PMMA) bars with diameter of $20 \mathrm{~mm}$ were used. Both incident and transmission bar had a length of $1750 \mathrm{~mm}$. One measurement point was placed on the incident bar, two measuring points were placed on the transmission bar. All measuring points were equipped with two pairs of strain-gauges (one pair semiconductor and one pair foil strain-gauges) in Wheatstone halfbridge arrangement. For this study, only data from foil strain-gauges 3/120 LY61 (HBM, Germany) with $3 \mathrm{~mm}$ active length were used since semiconductor strain-gauges exhibited non-linear behaviour in larger deformations and had a significantly reduced lifetime. The experiments were observed using a pair of synchronised high-speed camera Fastcam SA-Z (Photron, Japan) with resolution of $256 \times 168$ px at approx. $252 \mathrm{kfps}$. Sufficient illumination of the specimen surface was achieved using a pair of high intensity LED lights (Constellation 60, Veritas, USA). Note, that only one of the two cameras observed the surface of measured sample and its close proximity. Record from the second camera was not used for evaluation in this study but only for general inspection of the experiment. Overview of the used experimental setup and the basic principle of OHPB are shown in Fig. 3 

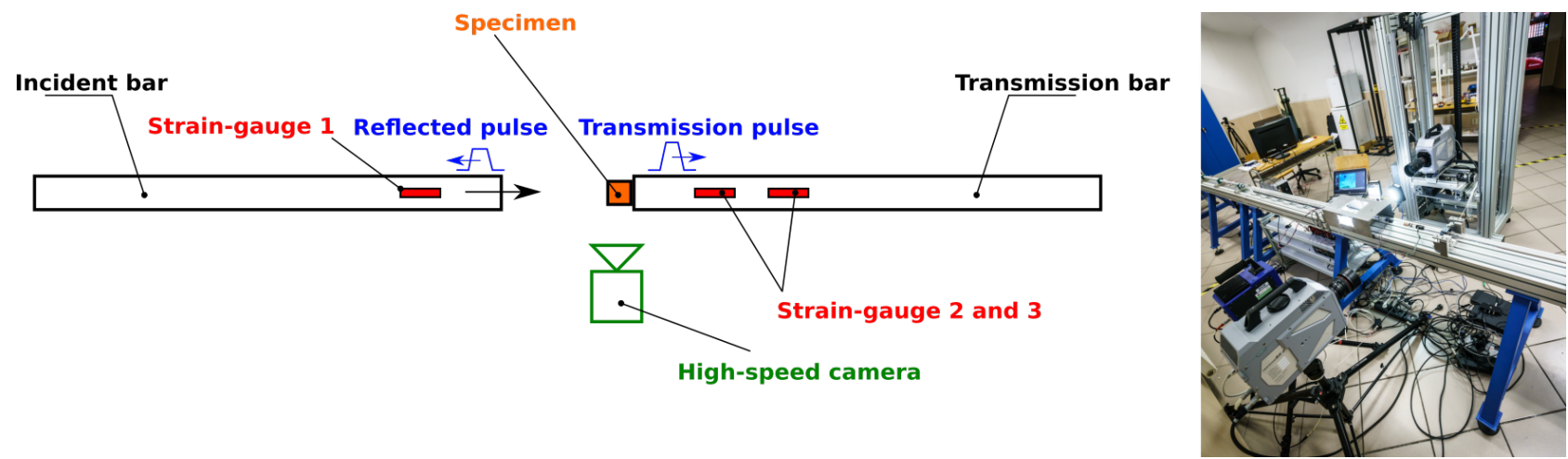

Figure 3. Principle of OHPB method with schematic of high speed camera used for DIC evaluation (left), Hopkinson Bar experimental setup with a pair o high-speed cameras (right)

\section{DIC TOOLS COMPARISON}

\subsection{FULL-FIELD DIC COMPARISON}

A virtual grid of $22 \times 14$ (columns $\times$ rows) correlation points was created to cover the area of the specimens surface and the ends of the measuring bars on the high-speed camera images. The same parameters of DIC evaluation were set for all three compared softwares: subset size $M=23 \mathrm{px}$, offset size $K=33$ px, grid spacing $N=4 \mathrm{px}$. Evolution of the full-field in-plane displacement of the representative auxetic specimen with $60 \mu \mathrm{m}$ layer of coating deformed using the OHPB apparatus and evaluated using the DIC by all three correlation softwares is shown in Fig. 6 . Correlation of high-speed camera images was possible only up to the 100th image in the sequence which corresponds to approx. $0.15-0.2$ overall strain (35 px of subset displacement). For higher displacement, NCorr provides distorted results due to low value of correlation coefficient and loss of correlation points. ISTRA4D and custom DIC tool results are reliable up to 130th image (approx. 0.25-0.3 overall strain, displacement of $45 \mathrm{px}$ ). Dynamic equilibrium was achieved during dynamic experiment as the sample was deformed uniformly on the both ends.

\subsection{DIC AND STRAIN-GAUGE RESULTS COMPARISON}

The stress-strain curves with strain calculated using the three DIC tools paired with the strain-gauge stress compared with the curve from conventional straingauge evaluation are shown in Fig. 44 DIC strain was for all software tools derived by tracking grid of correlation points created on the both edges of the deformed specimen. Stress-strain curves derived using all correlation tools are in a very good agreement with the strain-gauge curve on both samples up to 0.16 of overall strain (100th image). For ISTRA4D software and our custom correlation tool it is possible to perform correlation up to 0.25 of overall strain. Differences of the DIC curves to strain-gauge curve after 0.25 is due to large deformations of the individual cells of the tested structure resulting in a loss of correlation.

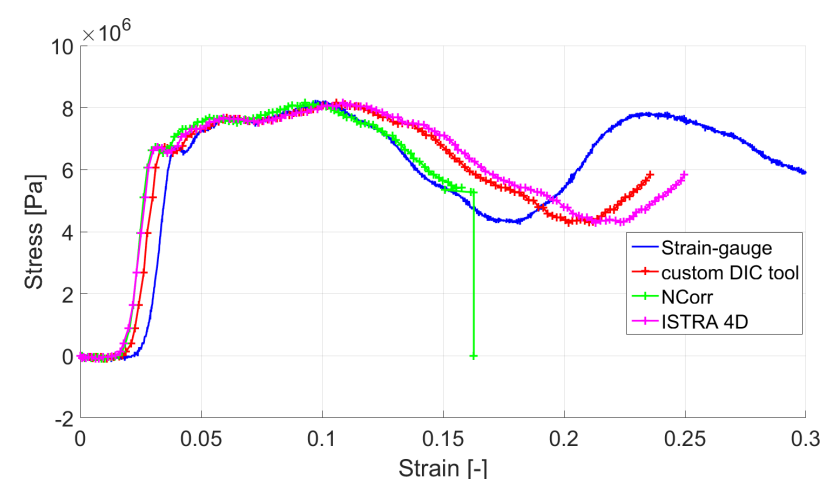

Figure 4. Comparison of stress-strain diagrams with strain evaluated from strain-gauges and DIC tools

\subsection{DIC SINGLE NODAL POINT ANALYSIS}

To further investigate the process of correlation of the virtual grid, the correlation of a single grid point located in the node of the specimen structure was performed. Tracked point of the structure and its location during the deformation is shown in Fig. 7 Original location of the point is marked by the green cross, the red cross marks the location evaluated from our custom tool and the blue cross from NCorr. Note, that for relatively small displacement, the location provided by both software tools is identical (Frame 30). The displacement of the same nodal point through the image sequence is shown in Fig. 5

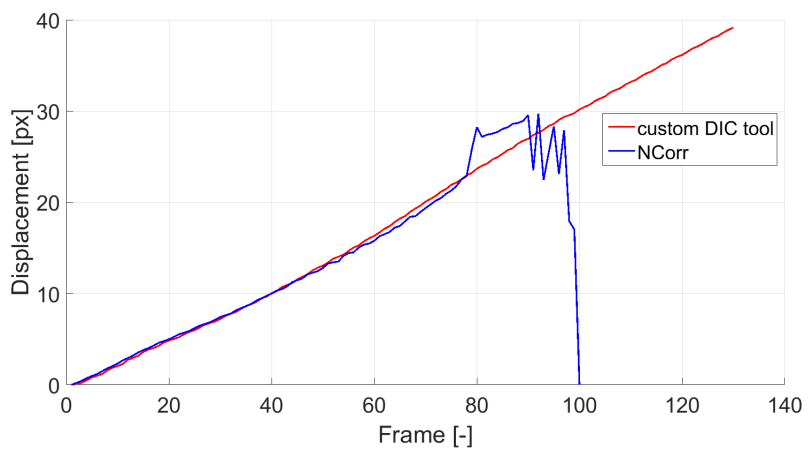

Figure 5. Displacement of single node point through image sequence 


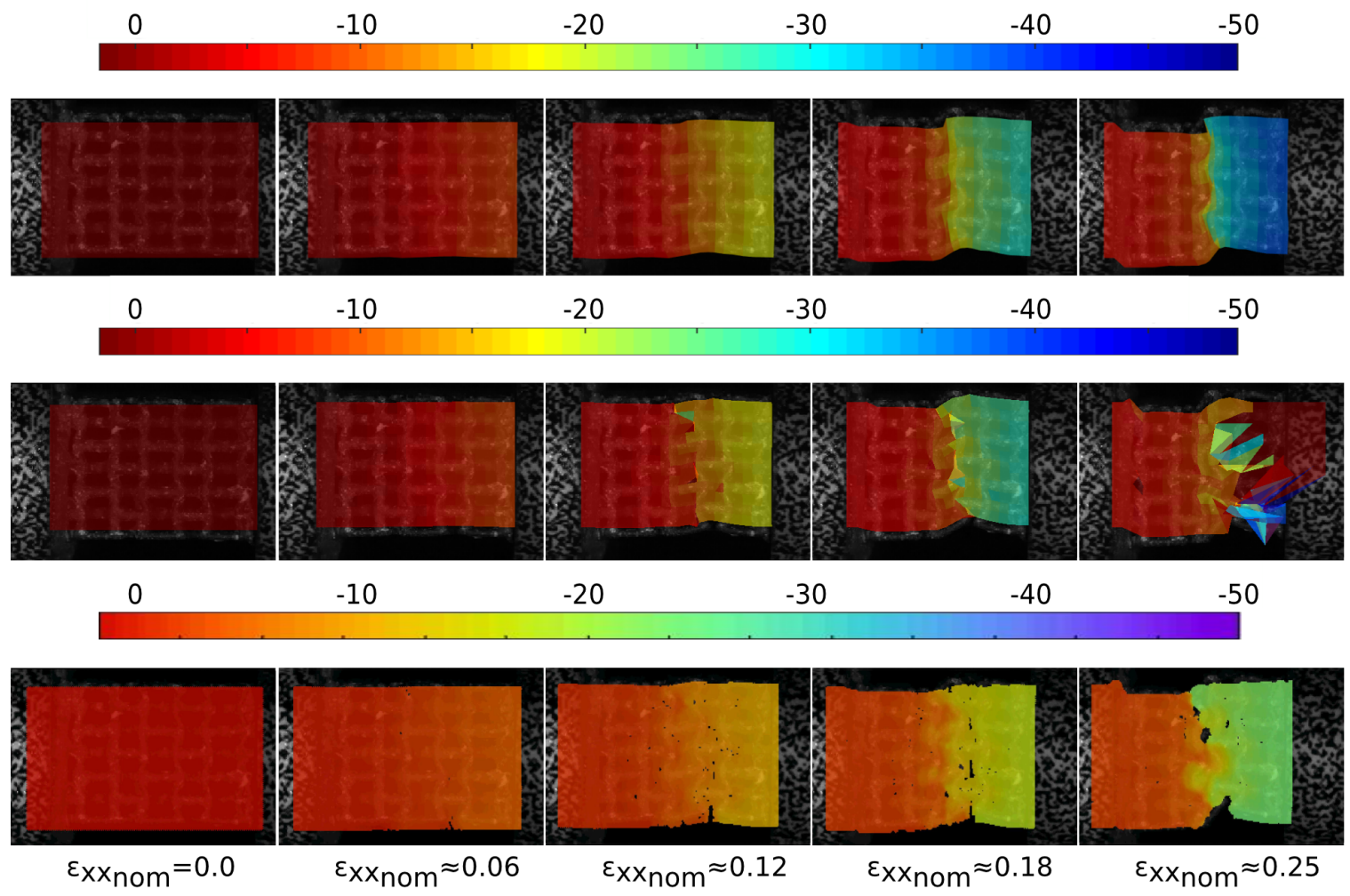

Figure 6. Full-field DIC analysis, OHPB measurement of auxetic structure: displacement in pixel, Custom DIC tool (top), NCorr (middle), ISTRA4D (bottom); note, that ISTRA4D uses different color range

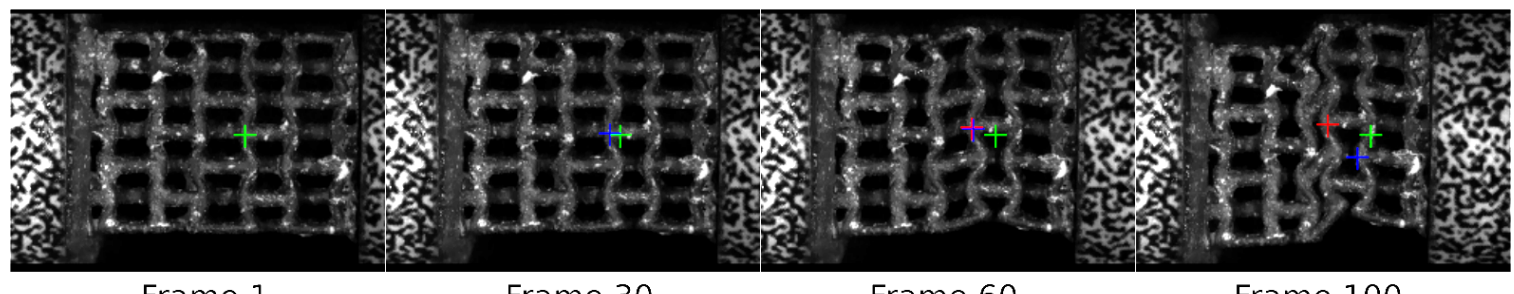

Frame 1

Frame 30

Frame 60

Frame 100

FiguRE 7. Single correlation point tracking, original point position (green), custom DIC tool (red), NCrorr (blue)

It is shown, that both the custom developed DIC tool and NCorr track the nodal point reliably up to the 80 th frame in the sequence. After that, the reliability of custom made software is much better. This is also illustrated by the graph shown in Fig. 8 . which shows the correlation coefficient mean value across all the correlation points in virtual grid. It is shown, that although the correlation criterions are different, the drop of correlation coefficient in NCorr software is much larger through the image sequence than in custom developed DIC tool.

\section{Conclusion}

OHPB measuring device with low impedance PMMA bars was successfully used for dynamic testing of the nickle coated 3D printed specimens of auxetic structure. Three different DIC tools were used for evaluation high-speed camera record of representative experiment. The results derived from all three software tools were compared and were found to be in a good agreement with the complementary strain-gauge

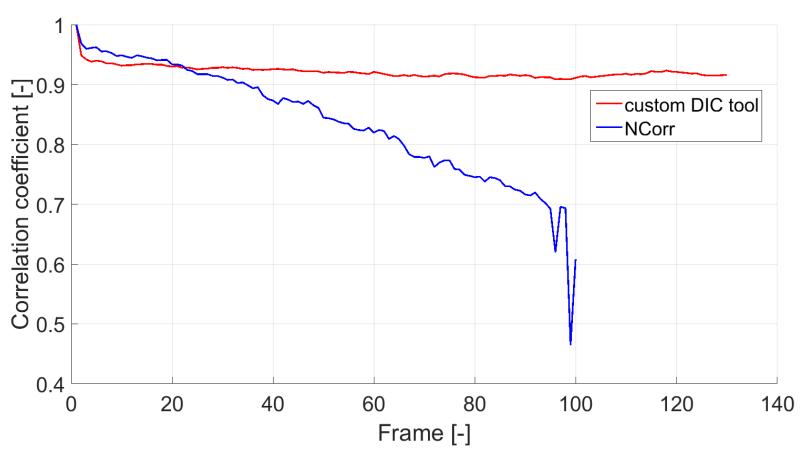

FiguRE 8. Correlation coefficient of compared DIC tools

measurement. The results from NCorr open-source tool were reliable up to $16 \%$ of deformation, the results from custom DIC tool and commercial ISTRA4D software were reliable up to $25 \%$ of deformation. Single nodal point analysis was carried out for further proving that our custom developed DIC software tool 
is not only comparable with other software tools but provides even more reliable results. It was shown that both, the OHPB and the DIC are suitable tools for advanced characterization of nickle coated auxetic structures under high strain-rate loading.

\section{ACKNOWLEDGEMENTS}

The research was supported by the Czech Science Foundation (project no. 19-23675S) and the internal grants of the Czech Technical University in Prague (projects no. SGS18/153/OHK2/2T/16 and SGS18/154/OHK2/2T/16). All the financial support is gratefully acknowledged.

\section{REFERENCES}

[1] T. Fíla, P. Koudelka, P. Zlámal, et al. Strain dependency of poisson's ratio of sls printed auxetic lattices subjected to quasi static and dynamic compressive loading, 2019. DOI:10.1002/adem.201900204

[2] I. Jandejsek, J. Valach, D. Vavřík. Optimization and calibration of digital image correlation method, 2010.
[3] J. Blaber, B. Adair, A. Antoniou. Ncorr: Open-source $2 \mathrm{~d}$ digital image correlation matlab software. Experimental Mechanics 55(6):1105-1122, 2015. DOI:10.1007/s11340-015-0009-1.

[4] A. Jung, M. Weinmann, H. Natter. Electroforming and Electrodeposition on Complex 3D Geometries: Special Requirements and New Methods, pp. 941-970. Springer International Publishing, 2016. DOI:10.1007/978-3-319-15266-0_46.

[5] B. D. Lucas, T. Kanade. An iterative image registration technique with an application to stereo vision. In Proceedings of the 7th International Joint Conference on Artificial Intelligence - Volume 2, pp. 674-679. Morgan Kaufmann Publishers Inc., San Francisco, CA, USA, 1981.

[6] R. A. Govender, R. J. Curry. The "open" hopkinson pressure bar: Towards addressing force equilibrium in specimens with non-uniform deformation. Journal of Dynamic Behavior of Materials 2(1):43-49, 2016. DOI:10.1007/s40870-015-0042-2 